\title{
Infrared Spectroscopy Beamline Based on a Tabletop Storage Ring
}

\author{
Md. Monirul Haque, ${ }^{1,2}$ Ahsa Moon, ${ }^{2}$ and Hironari Yamada ${ }^{2}$ \\ ${ }^{1}$ Department of Physics, Faculty of Science, University of Rajshahi, Rajshahi 6205, Bangladesh \\ ${ }^{2}$ Department of Photonics, Faculty of Science and Engineering, Ritsumeikan University, 1-1-1 Noji-higashi, \\ Kusatsu, Shiga 525-8577, Japan \\ Correspondence should be addressed to Md. Monirul Haque, mhpdru@yahoo.com
}

Received 22 February 2012; Accepted 29 April 2012

Academic Editors: A. E. Miroshnichenko and S. R. Restaino

Copyright ( $) 2012$ Md. Monirul Haque et al. This is an open access article distributed under the Creative Commons Attribution License, which permits unrestricted use, distribution, and reproduction in any medium, provided the original work is properly cited.

\begin{abstract}
An optical beamline dedicated to the infrared (IR) spectroscopy has been constructed at MIRRORCLE, a tabletop storage ring. The beamline has been designed for the use of infrared synchrotron radiation (IRSR) emitted from a bending magnet of $156 \mathrm{~mm}$ bending radius with the acceptance angle of $355(\mathrm{H}) \times 138(\mathrm{~V})$ mrad to obtain high flux. The IR emission is forced by an exactly circular optics, named photon storage ring (PhSR), placed around the electron orbit and is collected by a "magic mirror" associated with two plane mirrors in the storage ring. A Fourier transform interferometer has been installed to utilize the IRSR at MIRRORCLE. The design of the optical system and calculated results are reported.
\end{abstract}

\section{Introduction}

Synchrotron radiation (SR) is now a very promising candidate for the light source of infrared spectroscopy. SR is highly brilliant and directional. Several storage ring-based IR beamlines are operating around the world [1-4], and several others are under construction or are being planned $[5,6]$. Applications are multidisciplinary, and the number continues to increase as these beamlines become accessible to an increasing number of users. In particular, IR radiation from synchrotron radiation sources has found increasing use in research by means of Fourier transform spectroscopy especially in material and life sciences [7].

In the case of the infrared spectroscopy synchrotron radiation (IRSR) from a bending magnetic field, an ideal storage ring would be one equipped with the conditions of a large bending radius (for low divergence), a large stored current, a large aperture port (for high flux), and a low ring energy (for low heat load). In general, the third generation SR sources are equipped with large bending radius and are operated at high electron energies of 700-8000 MeV. Due to big size and high cost, they are incompatible in industry, hospitals, and research laboratories. Because of these shortcoming of the third-generation synchrotrons, the necessity of laboratorysized light sources have been discussed since the last decade.
Yamada proposed a far-IR laser by a tabletop synchrotron in 1989 [8]. Such a tabletop synchrotron has laid the foundation for the low-energy storage rings MIRRORCLE $[9,10]$, which is now a promising source for the use in a conventional laboratory or for clinical use.

MIRRORCLE-20 $[10,11]$ is a weak focusing type tabletop storage ring with a $156 \mathrm{~mm}$ radius exactly circular electron orbit. The configuration of this storage ring is listed elsewhere [12]. Because of this small orbit radius the stored beam current easily reaches ampere order by one-shot injection within $100 \mathrm{~ns}$ injection time window and $100 \mathrm{~mA}$ injector beam current. The elegance of MIRRORCLE technology lies in its conceptual simplicity. It has two basic components: classical microtron and a storage ring. The microtron emits and accelerates electrons up to a design level and then injects them into the storage ring. An IR beamline has been designed and constructed using this low energy synchrotron MIRRORCLE-20 exclusively dedicated to spectroscopy from mid-IR to far-IR regions. In this paper, we will present the designed optical system and simulated results.

\section{Front End}

Figure 1 shows a schematic view of the front end of MIRRORCLE-20 IR beamline. The optical system inside 


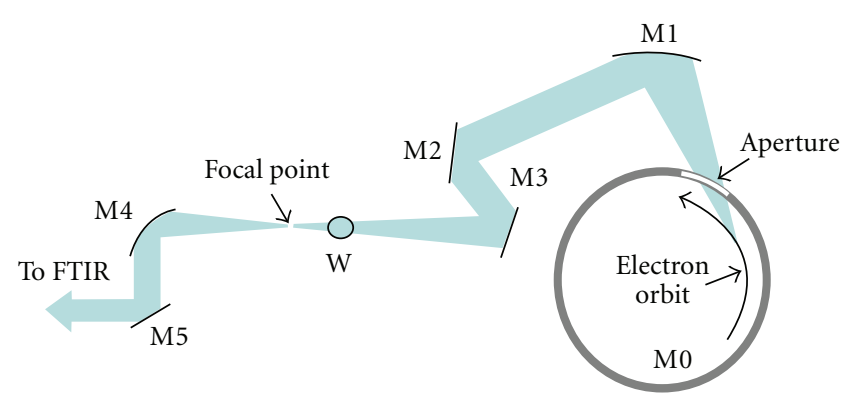

Figure 1: Schematic figure of the optical system of the infrared beamline at MIRRORCLE-20. M0, M1, and M4 indicate circular, magic and off-axis parabolic mirrors, respectively. M2, M3, and M5 are all plane mirrors. $\mathrm{W}$ is an optical window, which separates upstream ultrahigh-vacuum area and downstream low-vacuum one.

MIRORCLE-20 storage ring is somewhat different from usual SR beam optics. The special optical system is composed of an exactly circular concaved mirror (M0) concentric of the electron orbit, a magic mirror (M1), and two plane mirrors (M2 and M3). The circular mirror collects and accumulates SR photons emitted into $2 \pi$ of the electron orbit of length $1 \mathrm{~m}$ only. Yamada has named this optical system as a Photon Storage Ring (PhSR) [8]. The details about PhSR are described elsewhere [13]. An aperture on the circular mirror surface over 0.6 rad allows electron beam to be injected in as well as collected SR photons to be extracted out. The mirror M1, that is located at $290 \mathrm{~mm}$ from the center of the source, focuses SR photons from different position of electron beam trajectory, and mirrors M2 \& M3 direct the radiation to the first focal point. The magic mirror of width $160 \mathrm{~mm} \times$ height $40 \mathrm{~mm}$ can provide IR acceptance up to 355 (horizontal) $\times$ 138 (vertical) $\mathrm{mrad}^{2}$. The horizontal acceptance angle of $355 \mathrm{mrad}$ together with a bending radius $(156 \mathrm{~mm})$ of MIRRORCLE-20 gives an emission length of $0.06 \mathrm{~m}$.

Since the first focal point is located at $1.47 \mathrm{~m}$ from the focusing mirror (M1), it is difficult to get good focal conditions by using conventional spherical or elliptical mirrors. Therefore, we employed a three-dimensionally extended magic mirror proposed by López-Delgado and Szwarc [14]. The magic mirror not only focuses on the source but also reduces the time delay due to the large acceptance: the summation of the optical path length and the electron orbital length is the same for each position. The vertical direction is approximated by a spherical curve using the distance between the emission and focusing points and the incident angle to the magic mirror. The formulae of the coordinates of the surface of the magic mirror are

$$
\begin{aligned}
& F_{1}(\theta)=\frac{(1 / 2)\left\{\left(d_{0}-\rho \theta\right)^{2}-\rho^{2}-a^{2}\right\}+a \rho \sin \theta}{d_{0}-\rho \theta-a \cos \theta}, \\
& F_{2}(\theta)=\sqrt{\rho^{2}+F_{1}^{2}(\theta)+a^{2}-2 a\left(\rho \sin \theta+F_{1}(\theta) \cos \theta\right)},
\end{aligned}
$$

$$
\begin{aligned}
R(\theta) & =\frac{2 F_{1}(\theta)\left(d_{0}-\rho \theta-F_{1}(\theta)\right)}{d_{0}-\rho \theta} \\
& \times \cos \left[\frac{1}{2} \cos ^{-1}\left(\frac{F_{1}(\theta)-a \cos \theta}{F_{2}(\theta)}\right)\right], \\
x(\theta, v) & =\rho \cos \theta-F_{1}(\theta) \sin \theta \\
& +\frac{|R(\theta)|\left\{\sin \theta-\left(\rho \cos \theta-F_{1}(\theta) \sin \theta\right) / F_{2}(\theta)\right\}}{\sqrt{2+2\left(F_{1}(\theta)-a \cos \theta\right) / F_{2}(\theta)}} \\
& \times\left\{1-\cos \left(\tan ^{-1} \frac{v}{R(\theta)}\right)\right\}, \\
y(\theta, v) & =\rho \sin \theta+F_{1}(\theta) \cos \theta \\
& +\frac{|R(\theta)|\left\{-\cos \theta+\left(\left(a-\rho \sin \theta-F_{1}(\theta) \cos \theta\right) / F_{2}(\theta)\right)\right\}}{\sqrt{2+2\left(F_{1}(\theta)-a \cos \theta\right) / F_{2}(\theta)}} \\
& \times\left\{1-\cos \left(\tan ^{-1} \frac{v}{R(\theta)}\right)\right\}, \\
z(\theta, v) & =R(\theta) \sin \left(\tan ^{-1} \frac{v}{R(\theta)}\right),
\end{aligned}
$$

where $\rho$ is the electron orbit radius, $a$ is the length between the center of the electron orbit and the focal point, $d_{0}$ is the optical pass from a standard point, $\theta$ is the acceptance angle of SR from electron orbit, and $v$ is the height of the magic mirror. The parameters for MIRRORCLE-20 IR are $\rho=$ $156 \mathrm{~mm}, a=1588.2 \mathrm{~mm}, d_{0}=2200 \mathrm{~mm}, \theta=1.3 \sim 1.5 \mathrm{rad}$, and $v=-20 \sim 20 \mathrm{~mm}$. $R$ represents the vertical curvature of the magic mirror, and $F_{1} \& F_{2}$ indicate the projections of the source point and the focal point, respectively, on the vertical axis of the magic mirror.

\section{Ray Trace Simulation}

We simulated the beam profile at focal point by using the "ZEMAX" ray-trace program (http://www.focus-software .com/) and determined the optimum optical system. A special type of light source modeled by DLL (Windows Dynamic Link Library) was used in order to support the desired properties of MIRRORCLE type synchrotron. The sources were placed at different angles around in an orbit of radius $156 \mathrm{~mm}$. The power of each source was set at $30 \mu \mathrm{W}$. Toroidal objects consisting of rectangular surface were placed around the source orbit for circular optics. The horizontal and vertical curvatures of this optical system were set at $216 \mathrm{~mm}$ and $148 \mathrm{~mm}$, respectively. The magic mirror was placed at $290 \mathrm{~mm}$ from the center of the SR emission point, and the focal point is located at $1740 \mathrm{~mm}$ from the magic mirror.

The result of the ray trace at the focusing point is shown in Figure 2. The beam size at the focal point depends on the electron beam size and the emission angle of the IRSR. The emission angle also depends on photon energy. The beam Gaussian assumed in this simulation was $\sigma_{h}=$ $6 \mathrm{~mm}$ in horizontal direction, and $\sigma_{v}=3 \mathrm{~mm}$ in vertical direction. Figure 2 is the result at the photon energy of $0.1 \mathrm{eV}$ $\left(\sim 1000 \mathrm{~cm}^{-1}\right)$, a typical value of mid-IR region, and at the 


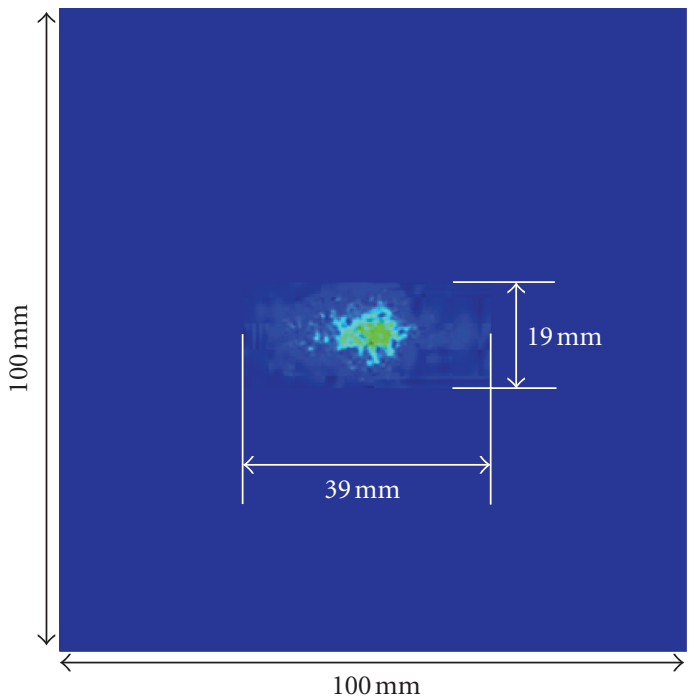

FIGURE 2: Ray trace beam pattern in mid-IR region (at $0.1 \mathrm{eV}$ ) at the focal point.

emission angle of $\pm 25 \mathrm{mrad}$. The beam size at the focal point is $39(\mathrm{~h}) \times 19(\mathrm{v}) \mathrm{mm}^{2}$. As the ratio of the distance between the SR emission point and the magic mirror to that between the magic mirror and the focal point is $1: 6$, the image at the focal point should be enhanced 6 times. We, therefore, observe from the simulation result that the focusing efficiency of the magic mirror is about $92 \%$.

\section{Photon Storage Ring (PhSR) Output}

Observation of coherent synchrotron radiation (CSR) from a single bunch traveling in a bending magnet [15-18] has encouraged Dr. Yamada to pay attention to the lasing scheme with PhSR. Here, the CSR denotes radiation emitted from a number of electrons in a bunch all in the same phase. It is known that CSR is dominant in the range of wavelengths comparable to or longer than the longitudinal bunch length. Under this condition, the electrons emit photons in phase and a single-cycle electromagnetic pulse results with intensity proportional to the square of the number of electrons in the bunch $[19,20]$.

Interference effect of SR between successive bunches is experimentally proved to be important [21]. Including the effect of CSR and the successive bunches, Yamada proposed the formalism for PhSR under the circular mirror $[9,22]$ as

$$
\begin{aligned}
P(\lambda) d \Omega d \lambda & =p(\lambda) h N_{e}[1+(N-1) F(\lambda)] G(\lambda) d \Omega d \lambda \\
& \cong p(\lambda) h N_{e}\left[1+N_{e} F(\lambda)\right] G(\lambda) d \Omega d \lambda,
\end{aligned}
$$

where $\rho(\lambda)$ is the incoherent synchrotron radiation power from one electron, $h$ the harmonic number, $N_{e}$ the number of electrons in the bunch; thus, $h N_{e}$ gives the beam current and $\rho(\lambda) h N_{e}$ gives the normal incoherent synchrotron radiation power in mrad at a certain bandwidth. $F(\lambda)$ is a form factor which is the Fourier transform of the longitudinal electron

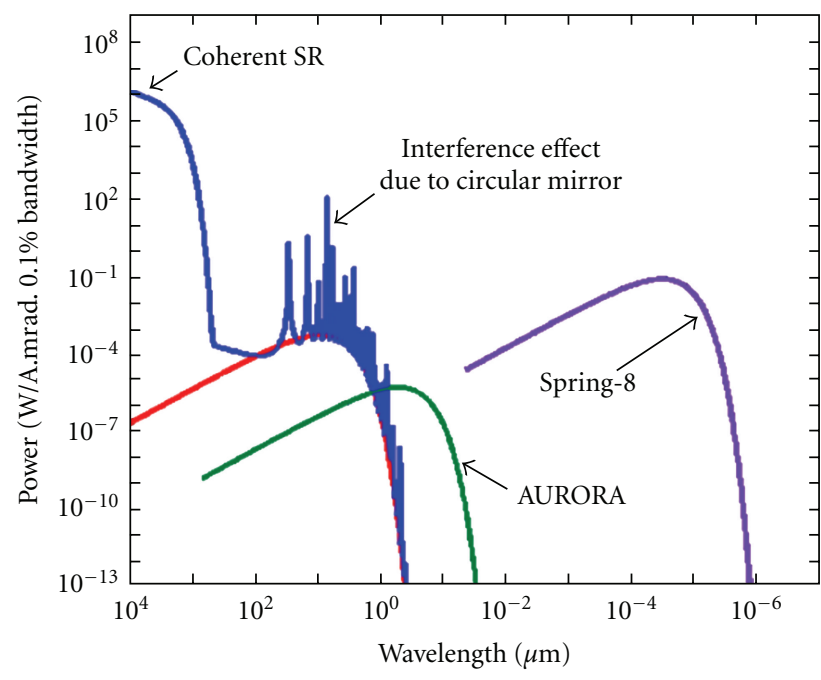

FIGURE 3: Spontaneous spectrum at 1A beam current and comparison with other beamlines, SR: Synchrotron Radiation.

distribution in the bunch. If a Gaussian electron distribution is assumed, $F$ is given as

$$
F(\lambda)=\exp \left[-\frac{1}{2}\left(\frac{2 \pi \sigma_{L}}{\lambda}\right)\right]
$$

where $\sigma_{L}$ is the r.m.s. bunch length. The function $G(\lambda)$ represents the interference between the coherent rays as well as between the incoherent rays as a result of the reflection due to the surrounding mirror (PhSR). This is given for a certain reflection coefficient $f$ as

$$
\begin{aligned}
G(\lambda) & =\left[\sum_{r}^{N_{b}}\left(f^{1 / 2}\right)^{r}\left\{\frac{\sin \left(r \pi \lambda_{R}\right)}{\sin \left(\pi \lambda_{R} / \lambda\right)}\right\}\right]^{2} \\
& \cong\left[\sum_{r}^{N_{b}}\left(f^{1 / 2}\right)^{r}\left\{\frac{\sin \left(\pi N_{i} \lambda_{R}\right)}{\sin \left(\pi \lambda_{R} / \lambda\right)}\right\},\right.
\end{aligned}
$$

where $N_{b}$ is the number of bouncing due to the circular mirror and $\lambda_{R}$ is the resonance wavelength. According to the above equations, the SR power in the case of $\mathrm{PhSR}$ is proportional to the square of the electron number, $N_{e}$ in the bunch, and to the square of the averaged number of interaction times, $N_{i}$. The resonant wavelength, $\lambda_{R}$ is given by

$$
\lambda_{R}=2\left(\theta+\frac{n \pi}{h}\right) \frac{\rho}{\beta}-2 \rho \cos \alpha \tan \theta,
$$

where $\alpha$ is the phase shift due to the beam size, $\beta$ is the normalized electron velocity, and $n$ is the integer indicating $n$ 'th electron bunch which merges with the light pulse.

The calculated spontaneous PhSR spectrum is shown in Figure 3. The calculation is based on the following conditions: beam current, $1 \mathrm{~A}$; bunch length, $0.5 \mathrm{~mm}$ in one standard deviation; power extraction efficiency, $30 \%$; resonance wavelength, $10 \mu \mathrm{m}$; average number of interaction, 100 . The calculated far-IR power of MIRRORCLE-20 is larger than the big machine Spring-8 [23] and AURORA [24]. The peaks are 
seen at the fundamental and higher harmonic wavelengths. Since the mirror is assumed to be truly circular, these higher harmonics should be seen up to very short wavelengths. However, depending on the tolerance of the mirror radius, it may not be possible to see these higher harmonics.

\section{Conclusions}

A new infrared beamline is presented at MIRRORCLE. The beamline is equipped with a Michelson-type FTIR for covering the spectral range $10-7800 \mathrm{~cm}^{-1}$. Ray-trace simulations using ZEMAX helped optimizing the optical parameters. The new beam line has higher power than other IR beamlines because of the new conceptual optics including the threedimensionally extended magic mirror with a large acceptance angle. We expect that the new beamline will provide us with a new opportunity to perform various kinds of experiments useful for broadband reflection and infrared spectroscopy.

\section{Acknowledgments}

This work was supported by the 21 st century COE program. The authors are very thankful to their numerous colleagues at MIRRORCLE as well as all members of PPL for their fruitful collaboration and discussion.

\section{References}

[1] R. P. S. M. Lobo, J. D. LaVeigne, D. H. Reitze, D. B. Tanner, and G. L. Carr, "Performance of new infrared beamline U12IR at the National Synchrotron Light Source," Review of Scientific Instruments, vol. 70, no. 7, pp. 2899-2904, 1999.

[2] S. Kimura, E. Nakamura, T. Nishi et al., "Infrared and terahertz spectromicroscopy beam line BL6B(IR) at UVSOR-II," Infrared Physics and Technology, vol. 49, no. 1-2, pp. 147-151, 2006.

[3] U. Schade, A. Röseler, E. H. Korte et al., "New infrared spectroscopic beamline at BESSY II," Review of Scientific Instruments, vol. 73, no. 3, p. 1568, 2002.

[4] Y. L. Mathis, H. O. Moser, and R. Steininger, "Status of and research opportunities at anka synchrotron radiation funder construction," Ferroelectrics, vol. 249, no. 1, pp. 11-20, 2001.

[5] P. Dumas, F. Polack, B. Lagarde, O. Chubar, J. L. Giorgetta, and S. Lefrançois, "Synchrotron infrared microscopy at the French Synchrotron Facility SOLEIL," Infrared Physics and Technology, vol. 49, no. 1-2, pp. 152-160, 2006.

[6] J. M. Byrd, M. C. Martin, W. R. McKinney et al., "CIRCE: a dedicated storage ring for coherent $\mathrm{THz}$ synchrotron radiation," Infrared Physics and Technology, vol. 45, no. 5-6, pp. 325330, 2004.

[7] W. B. Peatman and U. Schade, "A brilliant infrared light source at BESSY," Review of Scientific Instruments, vol. 72, no. 3, pp. 1620-1624, 2001.

[8] H. Yamada, "Photon storage ring," Japanese Journal of Applied Physics, vol. 28, no. 9, pp. 1665-1668, 1989.

[9] H. Yamada, "The smallest electron storage ring for highintensity far-infrared and hard X-ray productions," Journal of Synchrotron Radiation, vol. 5, no. 6, pp. 1326-1331, 1998.

[10] H. Yamada, "Novel X-ray source based on a tabletop synchrotron and its unique features," Nuclear Instruments and Methods in Physics Research B, vol. 199, pp. 509-516, 2003.
[11] H. Yamada, Y. Kitazawa, Y. Kanai et al., "Development of the hard X-ray source based on a tabletop electron storage ring," Nuclear Instruments and Methods in Physics Research A, vol. 467-468, pp. 122-125, 2001.

[12] A. Moon, N. Miura, H. Yamada, and M. M. Haque, "FIR beam line of MIRRORCLE-20," in Proceedings of the 2nd International Symposium on Portable Synchrotron Light Sources and Advanced Applications, vol. 902, pp. 23-25, January 2007.

[13] M. M. Haque, H. Yamada, A. Moon, and M. Yamada, "Performance of the far-IR beamline of the $6 \mathrm{MeV}$ tabletop synchrotron light source," Journal of Synchrotron Radiation, vol. 16, no. 2, pp. 299-306, 2009.

[14] R. López-Delgado and H. Szwarc, "Focusing all the synchrotron radiation ( $2 \pi$ radians) from an electron storage ring on a single point without time distortion," Optics Communications, vol. 19, no. 2, pp. 286-291, 1976.

[15] T. Nakazato, M. Oyamada, N. Niimura et al., "Observation of coherent synchrotron radiation," Physical Review Letters, vol. 63, no. 12, pp. 1245-1248, 1989.

[16] Y. Shibata, K. Ishi, T. Ohsaka et al., "Coherent synchrotron radiation at submillimeter and millimeter wavelengths," Nuclear Inst. and Methods in Physics Research A, vol. 301, no. 1, pp. 161-166, 1991.

[17] K. Ishi, Y. Shibata, T. Takahashi et al., "Spectrum of coherent synchrotron radiation in the far-infrared region," Physical Review A, vol. 43, no. 10, pp. 5597-5604, 1991.

[18] A. Renieri, "Storage ring operation of the free-electron laser: the amplifier," Il Nuovo Cimento B, vol. 53, no. 1, pp. 160-178, 1979.

[19] F. C. Michel, "Intense coherent submillimeter radiation in electron storage rings," Physical Review Letters, vol. 48, no. 9, pp. 580-583, 1982.

[20] C. J. Hirschmugl, M. Sagurton, and G. P. Williams, "Multiparticle coherence calculations for synchrotron-radiation emission," Physical Review A, vol. 44, no. 2, pp. 1316-1320, 1991.

[21] D. J. Wingham, "Electron synchrotron radiation in the far infrared," Physical Review D, vol. 35, no. 8, pp. 2584-2594, 1987.

[22] H. Yamada, "Current status and biological research ramifications of photon storage ring as a noble infrared laser source," Advances in Colloid and Interface Science, vol. 71-72, pp. 371392, 1997.

[23] S. Kimura, H. Kimura, T. Takahashi et al., "Front end and optics of infrared beamline at SPring-8," Nuclear Instruments and Methods in Physics Research A, vol. 467-468, pp. 437-440, 2001.

[24] T. Yaji, Y. Yamamoto, T. Ohta, and S. Kimura, "A new beamline for infrared microscopy in the SR center of Ritsumeikan University," Infrared Physics and Technology, vol. 51, no. 5, pp. 397-399, 2008. 

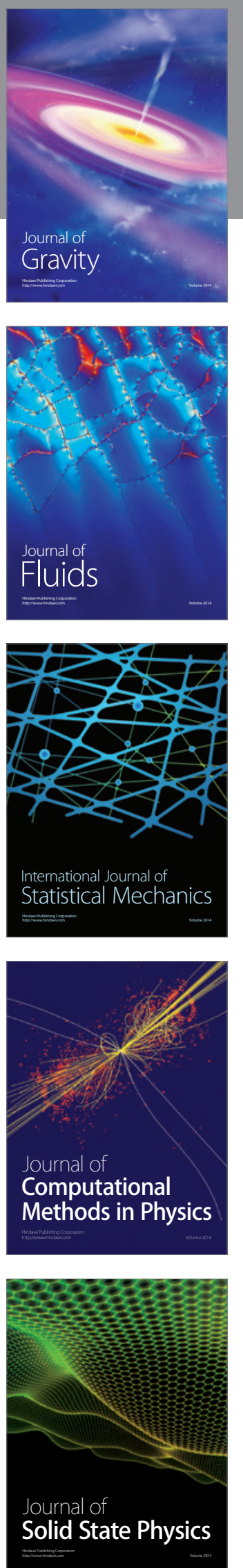
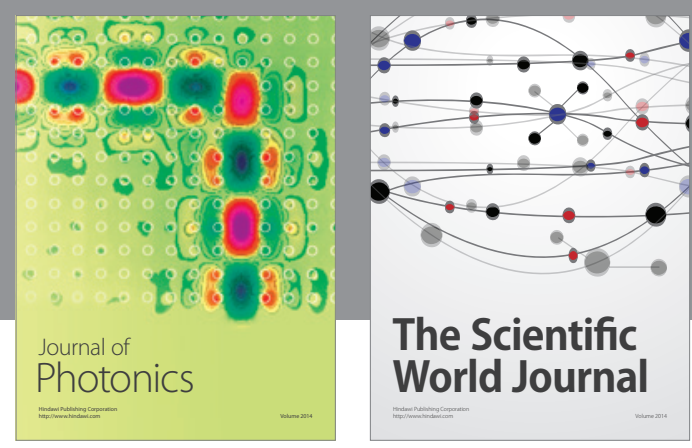

The Scientific World Journal

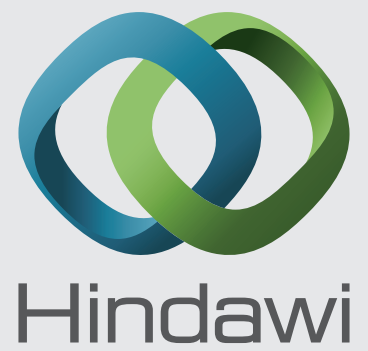

Submit your manuscripts at http://www.hindawi.com
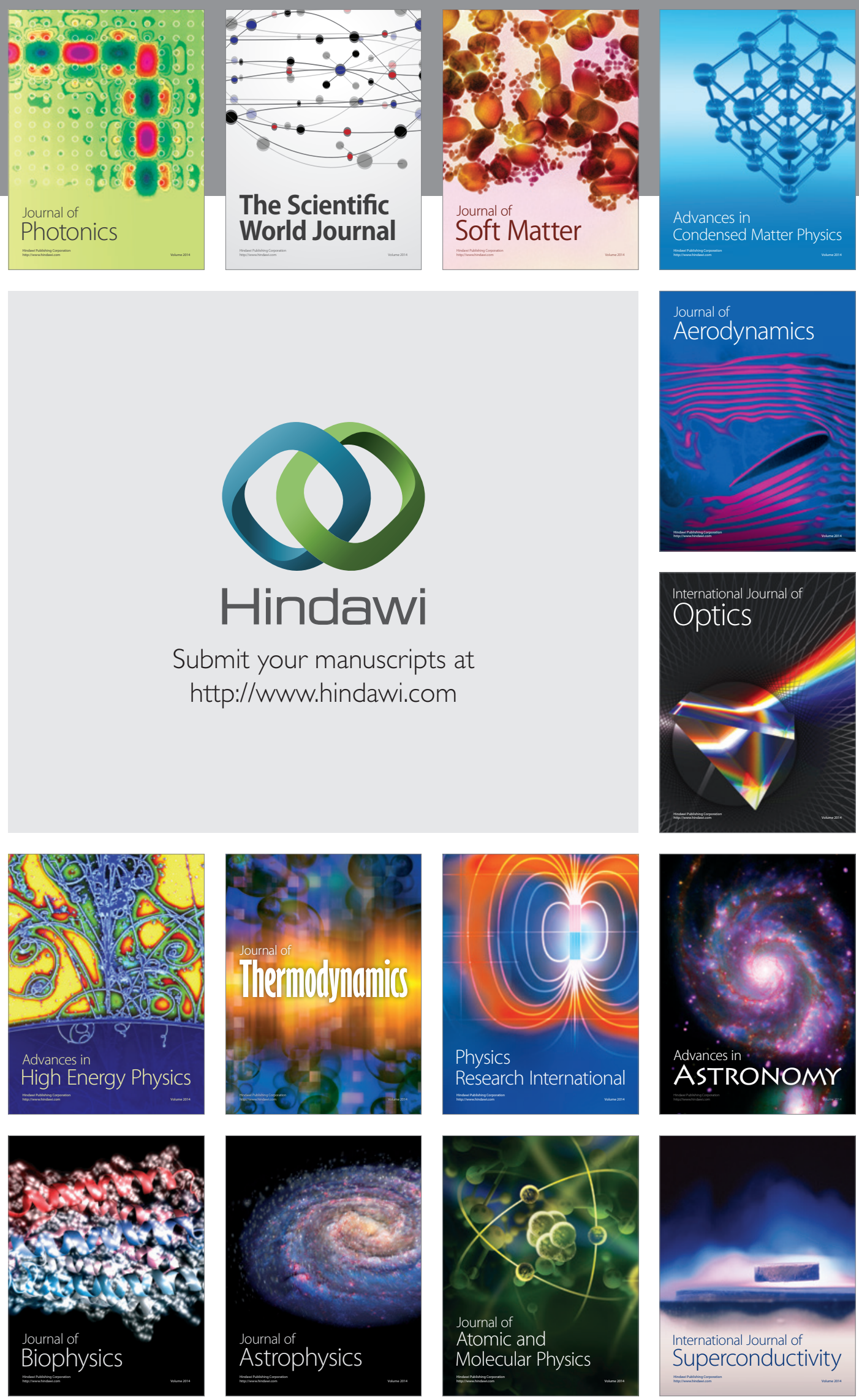
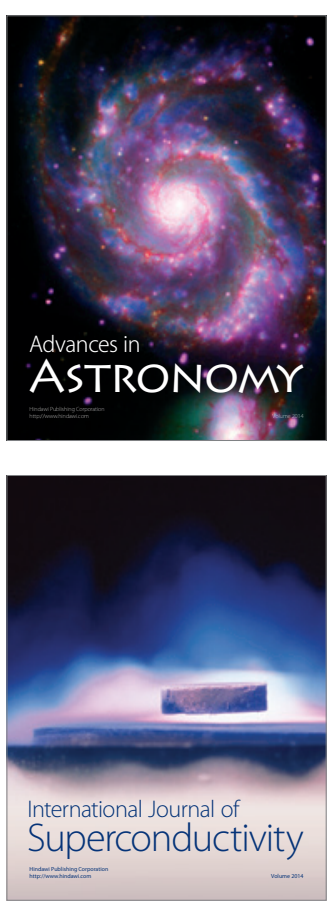